Address for Correspondence: Dr. Jian Kang,

Department of Respiratory Disease and Internal Medicine, Shengjing Hospital,

China Medicine University, Shenyang

110004, Liaoning Province, China.

E-mail: kangjian58@163.com

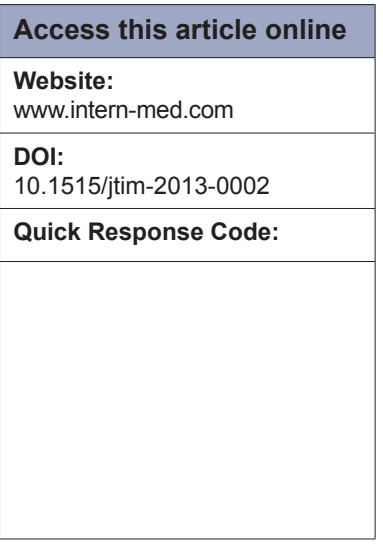

\title{
Journal of Translational Internal Medicine: Birth at the right moment
}

\author{
Jian Kang
}

Department of Respiratory Disease and Internal Medicine, First Affiliated Hospital of China Medical University, Shenyang 110004, Liaoning Province, China

At the beginning of modern medicine, namely, from the 19th century to the first half of 20 th century, internal medicine had always been an integral discipline. The physicians at that time scorned to be specialists who only treated specific diseases. With the development of basic sciences, increasingly fine clinical medicine, the gradually elevated demands for better medical service from patients, the effects of the industrial division on the working model of physicians, etc., have contributed to the rapid growth and development of specialties within internal medicine. Now that aging approaches, internal physicians who can handle a variety of diseases, rather than specialists, are preferred by senile patients. The establishment of internal medicine can better assist hospitals in the management of and coordination between specialties. Moreover, general internal medicine, which shoulders the chief responsibility of expanding interdisciplinary fields of medicine, has established close ties to Emergency department, ICU and community hospitals. Experience has shown that to expand general internal medicine in large general hospitals would virtually be the return of internal medicine. In summary, internal medicine has an irreplaceable position, irrespective of either academic settings, hospital management, or patients' needs and personnel training. ${ }^{[1]}$ Only strong internal medicine can ensure the healthy and sustainable development of specialty. Emphasis has been placed on the development of internal medicine. To establish general internal medicine is probably a correct path.

The concept of translational medicine, which appeared for the first time in Lancet in 1996, refers to the rapid and efficient transformation process of basic biological research clinical technology, namely, from laboratory to clinic, then again from clinic to laboratory. ${ }^{[2]}$ Currently, the concept of translational medicine, which has been further expanded, represents the synergy between epidemiology, basic research and clinical trials, and is based upon Innovation Management and Research Development in medicine. ${ }^{[3]}$ So far, translational medical centers have been established in over 40 universities across the U.S., and its number is expected to reach 80 or more in 2013. Annual funding for translational medical research reaches $\$ 500$ million by NIH, $£ 450$ million in U.K., and up to 6 billion euros by the European Community. In addition, some renowned medical journals have set up translational medicine-related columns and professional journals, such as Science Translational Medicine, Journal of Translational Medicine and Translational Research. ${ }^{[4]}$ Research articles on translational medicine with an increasing number have received increasing attention from medical research scholars. Translational medical research actually has assumed the macro role of disease control, integrating application of the results of basic research, solutions to problems encountered in clinical practice, and implementation of disease prevention. To sum up, demand of practical disease control is the driving force for the development of translational medicine.

Experiences with Disease Control from other countries show that for most diseases, prevention is the most important yet the most economical step in disease control. Introduction of disease prevention into translational medicine, therefore, is inexorably critical to disease control. 
Cardiovascular research conducted in the 1960s and 1970s in U.S. has found that tobacco control can reduce cardiovascular risk and have obtained some results. Moreover, translational research regarding community diabetes control in Australia has shown that community disease prevention projects, coupled with translational research designs, are easier to implement, and its measures are more effective, resulting in increased output. Translational medicine is actually a more effective research form. It efficiently promotes research development through effective information gathering and circulation. A promising translational medical research requires reasonable disease selection, and research process demonstration which confirms that such research could promote the control of such disease. Generally, diseases greatly harmful to people should be listed as first choice of research. Currently, tumor, infectious diseases and diabetes are most studied in translational research. ${ }^{[5-7]}$

To reorganize research currently available is an effective way as well to conduct translational research. For instance, academic conferences provide opportunities for people to discuss its research progress and disease control who are committed to basic research, clinical practice and prevention of the same disease, so that further actual integrated work could be conducted. To understand the job content of different levels and disease progress through communication and exchange of researchers so as to facilitate the development and control of disease also falls into the scope of translational medicine. Multi-center translational medical research: In the case of a rational selection of research subject matter, conduction of multicenter research can accelerate the research process. Multicenter cooperation and coordination are critical to the success of the multi-center translational medicine. Research managers should take into consideration heterogeneity of different centers in the study design stage, for instance, composition of patients, comparability of test methods, and consistent requirements for data collection in different centers. To achieve this goal, generally, people with multicenter experience are needed to develop standardized work procedures. Project manager of translational medicine should command basic characteristics of various fields involved in research, and be able to coordinate and make arrangements accordingly. Of course, some translational medical researches are conducted independently by workers in the field of application. Such researchers must acquire knowledge and skills of disease prevention both at the macro- and micro- levels. For this reason, in terms of education, it is necessary to introduce basic knowledge of medicine-related fields to medical students, to enhance their competence to communicate and organize and to strengthen their understanding of disease prevention and control as well..$^{[8]}$
Thus, translational medicine is an inevitable trend along with medical advances. In addition, with strengthened holism of disease prevention and treatment, as well as higher individual requirements for health, multi-center even worldwide corporation on the same disease is likely to become a reality. Moreover, currently, large translational medical research will be the direction of development for local translational medical research. Additionally, such research would cover more disease prevention. And to improve the health of all mankind would be its ultimate goal and its core. Translational medical research all over the world has a good momentum of development, thus research management should provide macro- guidance for a rationally expanded research so as to promote the development of translational medicine in China and to improve the output level of research. ${ }^{[0]}$

Rapid development of internal medicine as well as translational medicine, actually urgently needs a journal to host their scientific output. For this reason, with the advent of Christmas 2013, the Journal of Translational Internal Medicine has finally arrived. This journal has come into being as it' supposed to. The aim of the journal is to rapidly report the new progress, new insights on translational internal medicine and those related to translational internal medicine, and to publish the related articles, reviews, expert forum, guidelines, etc. All aspects of internal medicine will be covered, including Respiratory diseases, Circulatory diseases, Gastroenterology, Urinary diseases, Hematology, Kidney diseases, Endocrinology, etc. The mission of the journal is to construct a platform of integrating basic medicine and clinical medicine. Based on interdisciplinary and disciplinary integration, with the guideline of exchange of ideas, academic discussions, innovation and inspiration, this journal provides a professional platform for academic exchanges amongst a majority of scholars, and related medical workers in internal medicine. Here, on behalf of editors and expert team of translational internal medicine, I would like to extend my sincere gratitude to my colleagues and friends supportive of our journal, and also wish a better tomorrow for our journal!

\section{REFERENCES}

1. Shen T. To construct and develop internal medicine in the trend of specialization. Pek Union Med J 2010;1:119-20.

2. Geraghty J. Adenomatous polyposis coli and translational medicine. Lancet 1996;348:422.

3. Polese F, Capunzo M. The determinants of translational medicine success - A managerial contribution. Transl Med UniSa 2013;6:29-34.

4. Tian L, Zhang HL, Ma LF. Current status and prospect of translational medicine home and abroad. J Med Res 2011;40:17-20.

5. Tuveson D, Hanahan D. Translational medicine: Cancer lessons from mice to humans. Nature 2011;471:316-7. 
6. Fears R, van der Meer JW, ter Meulen V. Translational medicine policy issues in infectious disease. Sci Transl Med 2010;2:14cm2.

7. Shah IM, Mackay SP, McKay GA. Therapeutic strategies in the treatment of diabetic nephropathy - A translational medicine approach. Curr Med Chem 2009;16:997-1016.

8. Wang YL, Wang YJ. Translational Medicine Research and Practice of cerebrovascular disease in Tiantan Hospital - Out of the concept. Transl Med Res 2011;1:8-16.
9. Li AJ, Sun YL, Cheng QB, Yang MH, Cheng CM. Translational Medicine and research hospitals. Chin Hosp 2013;17:10-2.

How to cite this article: Kang J. Journal of Translational Internal Medicine: Birth at the right moment. J Transl Intern Med 2013; 1(1): 3-5. Source of Support: Nil, Conflict of Interest: None declared 